\title{
Using informative features in machine learning based method for COVID-19 drug repurposing
}

Rosa Aghdam ${ }^{1 *+}$ (I), Mahnaz Habibi ${ }^{2+}$ and Golnaz Taheri ${ }^{3,4+}$

\begin{abstract}
Coronavirus disease 2019 (COVID-19) is caused by a novel virus named Severe Acute Respiratory Syndrome Coronavirus-2 (SARS-CoV-2). This virus induced a large number of deaths and millions of confirmed cases worldwide, creating a serious danger to public health. However, there are no specific therapies or drugs available for COVID-19 treatment. While new drug discovery is a long process, repurposing available drugs for COVID-19 can help recognize treatments with known clinical profiles. Computational drug repurposing methods can reduce the cost, time, and risk of drug toxicity. In this work, we build a graph as a COVID-19 related biological network. This network is related to virus targets or their associated biological processes. We select essential proteins in the constructed biological network that lead to a major disruption in the network. Our method from these essential proteins chooses 93 proteins related to COVID-19 pathology. Then, we propose multiple informative features based on drug-target and protein-protein interaction information. Through these informative features, we find five appropriate clusters of drugs that contain some candidates as potential COVID-19 treatments. To evaluate our results, we provide statistical and clinical evidence for our candidate drugs. From our proposed candidate drugs, $80 \%$ of them were studied in other studies and clinical trials.
\end{abstract}

Keywords: Coronavirus disease 2019, SARS-CoV-2, Protein-protein interaction, Clustering method

\section{Introduction}

The pandemic situation for Coronavirus disease 2019 (COVID-19) causes more than 197 million infections and more than 4.2 million deaths in more than 200 countries worldwide (until the end of July 2021) and this number is increasing rapidly. Due to this rapid spread, researchers have been searching for therapeutic approaches in the past few months. At present, no medicine has been claimed to be effective in the treatment or even prevention of COIVD-19 [1]. On the other hand, producing new drugs with a complete drug profile is a tough task that

\footnotetext{
*Correspondence: rosa.aghdam@ipm.ir

${ }^{\dagger}$ Rosa Aghdam, Mahnaz Habibi and Golnaz Taheri have contributed equally to this work

${ }^{1}$ School of Biological Sciences, Institute for Research in Fundamental Sciences (IPM), Tehran, Iran

Full list of author information is available at the end of the article
}

requires extensive time and budget. Drug repurposing is the procedure of perusing new therapeutic uses for available drugs. This process can reduce a large amount of time, money, and danger of the traditional drug discovery process [2]. The main purpose of drug repurposing is to exceed the therapeutic use of the available drugs for more medical scope. Previous researches showed that drugs with similar profiles probably demonstrate similar behavior in the existence of similar targets like proteins [1-3]. Traditional drug repurposing methods are mainly based on finding the relationship between biological activity and the molecular structure of different drugs. However, newer data gathering and analysis shows the urgent need for using computational methods for drug design and repurposing. Computational methods are mainly used to discover different drug interactions that are not considered and found during the clinical trial process [4]. In 
drug repositioning, computational methods investigate the relationship between drug databases and genomic, transcriptomic, and other available information with the help of data and network analysis and machine learning methods [2]. Machine-learning based methods for drug repurposing reveal the connection between drugs, viral, and host proteins. In the life cycle of a virus, the viral proteins are associated with different human proteins in the infected cells through different interactions. Within these interactions, the virus hijacks the host cells for replication, and this process changes the regular function of these interacted proteins. Therefore, to design antiviral drugs, a complete understanding of the interaction between human proteins and viral is crucial [5]. It is worth mentioning, in drug repurposing to fight the virus, targeting just virus proteins is not the proper approach. Targeting single virus proteins can cause the viruses to escape this attack through some backup pathways. These backup pathways lead to increased virus resistance with the mutation. Host-directed treatments propose significant strategies [6]. These methods select human proteins as the main carriers for the virus to enter and control human cells. These host-directed treatments seem to be less susceptible to making resistance because human proteins are less influenced by mutations. Therefore, targeting human proteins as drug targets is a more sustainable strategy. In host-directed treatments, it is important to find proteins that are essential for the maintenance and persistence of the disease that is caused by a virus in the human cells. When these proteins are targeted as drug targets, the replication mechanism of the virus collapses. For all of the above-mentioned reasons, repurposing drugs with host-directed treatments against COVID19 has major potential. Furthermore, drug repurposing methods provide hope for fast practical implementation with the minimum side effects. Molecular interaction and biological interaction networks as valuable resources are the foundation for drug repurposing methods [7]. This means that network-based drug repurposing methods propose novel opportunities for finding drug targets in host-directed treatments [8]. Recent studies show that valuable results are based on viral-host networks for treating HIV [9], Hepatitis C [10], and Ebola as well [11]. Since the outbreak of COVID-19 some research groups have been trying to develop network-based methods to find some repurposed drugs to operate against SARS-CoV-2. Zhou et al. [12] proposed a network-based method for the identification of some candidates as repurposable drugs and some potential drug combinations targeting. Li et al. [13] combined network data with a relative analysis of the gene sequences of the different viruses to find potential drugs for SARS-CoV-2. Gordon et al. [14] proposed a map from human proteins with
SARS-CoV-2 proteins that were found to interact in the affinity purification mass spectrum method. Dick et al. [15] recognized high confidence interactions between human proteins and SARS-CoV-2 proteins with the help of sequence-based protein-protein interaction (PPI) predictors.

In this paper, we propose the four steps method. This method tries to identify novel drug targets and pathways associated with essential proteins in COVID-19. In the first step, we build a graph as a COVID-19 related biological network related to virus targets or their associated biological processes. In the second step, we use two effective algorithms $[16,17]$ to find the candidate set of proteins from biological networks that lead to a major disruption in the network. In the third step, we identify proteins in our candidate set that are associated with some underlying diseases related to COVID-19. Then, we select 93 proteins as a final set of essential proteins related to disease pathology. Identifying essential proteins may elucidate new drug targets and pathways related to COVID-19. In the fourth and last step, we propose informative features based on drug-protein and PPI networks and find five significant clusters that contain appropriate candidate drugs. Our results show that using our four steps method suggests some appropriate candidate drugs. Most of these candidate drugs are recommended in other studies.

\section{Methods \\ Finding essential proteins related to COVID-19 pathology as candidate drug targets}

Introducing the essential proteins related to COVID-19 pathology as candidate drug targets is one of the most used and appropriate ways to find suitable drugs for COVID-19 treatment. In this subsection, we describe the first, second and third steps of our proposed method. These two steps try to find the set of essential proteins related to COVID-19 pathology. In the first step, we use two effective algorithms $[16,17]$ for finding the minimum number of proteins that participate in a large number of biological processes. We use these algorithms to find sets of essential proteins based on the disruption of the COVID-19 related biological network. In the second step, we investigated COVID-19 associated protein sets. As a result of this step, we found a subset of essential proteins that are essential to disease pathology.

\section{Construction of COVID-19 related biological network}

Suppose that informative biological processes (IBP) is a set of biological processes related to virus targets in COVID19 that will be described in the next subsection. Two proteins are functionally interacted if they are connected through the same biological processes. A COVID-19 
related biological network is considered as a weighted undirected graph $G=(V, E, \omega)$. In this graph, each node $v_{i} \in V$ represents the protein and each edge $e_{i j} \in E$ represents a functional interaction between two nodes $v_{i}$ and $v_{j}$. The $\omega\left(e_{i j}\right)$ shows the weight of $e_{i j}$ that demonstrates the number of biological processes that two nodes $v_{i}$ and $v_{j}$ participate in them. A path between two nodes $v_{j}$ and $v_{k}$ in the graph is a sequence of edges that connect the number of distinct nodes through this path. In the weighted graph, the weight of the path between two nodes is defined as follows. Suppose that $v_{j}$ and $v_{k}$ as the two ends of this path. Then, the sum of the weight of edges between these two nodes is the weight of this path. A path with the minimum weight between these two nodes is named the shortest path. Now, we define the betweenness value for each node, $v_{i}$, in the graph in the following way:

$$
\operatorname{Betw}\left(v_{i}\right)=\sum_{v_{j}, v_{k} \in V} \frac{\theta_{e_{j k}} v_{i}}{\theta_{e_{j k}}},
$$

where $\theta_{e_{j k}}$ shows the total number of shortest paths from node $v_{j}$ to node $v_{k}$ and $\theta_{e_{j k}} v_{i}$ indicates the number of shortest paths that pass through node $v_{i}$.

\section{Disruption of COVID-19 related biological processes}

We adapt two algorithms to detect the essential proteins in the COVID-19 related biological network [16, 17]. These algorithms $[16,17]$ select some of the best candidates as removal proteins set from the COVID-19 related biological network to make a major disruption in it. We place the outputs of Algorithm 1 and 2 in $C u t_{1}$ and $C u t_{2}$, respectively.

\section{Algorithm 1: spectral partitioning}

Partitioning a simple graph, $G$, into disjoint balanced or nearly balanced parts with removing the minimum number of edges between these two parts is known as the NP-complete problem [16]. We try to approximate this partitioning problem with the spectral partitioning algorithm. This algorithm is based on eigenvectors of the Laplace of the graph, $G$, and divides the graph into two disjoint parts with respect to eigenvectors of a Laplacian matrix. It is worth mentioning that, the spectral partitioning algorithm is one of the best heuristic approaches for graph partition. Let $A=\left[a_{i j}\right]$ shows the adjacency matrix of graph $G$ such that,

$$
a_{i j}= \begin{cases}1 & \text { if }\left(v_{i}, v_{j}\right) \in E \\ 0 & \text { otherwise }\end{cases}
$$

We define a diagonal degree matrix $D=\operatorname{diag}\left(d_{i}\right)$ for graph $G$. In this matrix value $d\left(v_{i}\right)$ shows the degree of $v_{i}$ in graph $G$. The Laplacian matrix of the graph $G$ is defined by $L=D \backslash A$ and $L(G)=\left[l_{i j}\right]$ where,

$$
l_{i j}= \begin{cases}1 & \text { if }\left(v_{i}, v_{j}\right) \in E \\ d\left(v_{i}\right) & \text { if } i=j \\ 0 & \text { otherwise }\end{cases}
$$

The Laplacian matrix is a symmetric positive semi-definite matrix. This matrix has some important properties. Suppose that vector $u=\left(u_{1}, u_{2}, \ldots, u_{n}\right)$ shows the normalized eigenvectors of matrix $L(G)$ and vector $\left(\lambda_{1}, \lambda_{2}, \ldots, \lambda_{n}\right)$ demonstrates the corresponding eigenvalues of these eigenvectors. We first compute the eigenvectors of Laplacian matrix $L(G)$, according to the second smallest eigenvalue of this matrix,$\lambda_{2}$, and put them in vector $X=\left(x_{1}, \ldots, x_{n}\right)$. Then, we sort the elements of vector $X$ and insert half of the nodes in partition $G_{1}$ and the reminder of nodes in another partition $G_{2}$. This procedure divides the nodes of graph $G$ into two partitions, $G_{1}$ and $G_{2}$ with nearly equal sizes. Removing the edges between these two parts through the cut edges $E\left(G_{1}, G_{2}\right)$ makes these two parts disconnect. Suppose the vector $A=\left\{\alpha_{1}, \ldots, \alpha_{m}\right\}$ shows the vertices placed in part $G_{1}$ and vector $B=\left\{\beta_{1}, \ldots, \beta_{m}\right\}$ shows the vertices are placed in part $G_{2}$, respectively. To make these two parts, $G_{1}$ and $G_{2}$ disconnect, we choose vertices from vectors $A$ and $B$ repeatedly. The vertices are chosen with respect to their degrees and removed until the all edges in $E\left(G_{1}, G_{2}\right)$ are covered.

\section{Algorithm 2: betweenness value}

This algorithm [17] tries to make the maximum disruption in the network by removing the minimum number of essential proteins. The selection method in algorithm [17] is based on the betweenness value mentioned in Eq 1. The algorithm [17] has three parts. In the first part, the betweenness value for each node in the graph $G$ is calculated. In the second part, to separate the graph $G$ into two disjoint partitions $G_{1}$ and $G_{2}$, the node with the minimum betweenness value in graph $G$ is selected and placed in partition $G_{1}$. Then, from all of the neighbors of the selected node, the node with the minimum betweenness value is selected and placed in the other partition $G_{2}$. These procedures are repeated until all nodes are placed into two disjoint partitions $G_{1}$ and $G_{2}$. In the third part, the minimum number of nodes from two constructed partitions $G_{1}$ and $G_{2}$ is selected with respect to their betweenness values to remove all edges in $E\left(G_{1}, G_{2}\right)$. The third step of this algorithm is equivalent to the minimum bi-section problem that is an NP-complete problem [18].

\section{Candidate essential proteins associated with COVID-19 pathology}

COVID-19 is a pandemic disease and has different severity and symptoms for various patients. The severity of this disease can vary from asymptomatic to fatal for 
different people. Recent studies show that this disease has high severity in people with some underlying conditions. Some of the most related underlying diseases are Diabetes, Cardiovascular diseases, Lung diseases, Hepatitis, Kidney disease, and different types of cancer. Hence, we expect that the genetics of these underlying diseases has some correlations with the essential proteins in COVID-19. For finding these essential proteins, we use the relation between gene and disease from Database for Annotation, Visualization, and Integrated Discovery (DAVID). Then, we select some proteins through our two mentioned algorithms that are annotated to four out of five of these specific comorbid diseases. From these selected proteins, proteins with significant $\mathrm{p}$-values as a set of essential proteins associated with COVID-19 are chosen and placed in $E$ as a set of main target candidates of COVID-19 drugs.

\section{Drug clustering method}

\section{Protein-protein interaction network}

We use 5 human high-throughput PPI networks in this work. The first one, Huri, contains 52,248 binary interactions [19]. The second one is collected from the biological general repository for interaction datasets (BioGRID) and contains 296,046 interactions [20]. The BioGRID dataset contains various interactions that are created from different techniques. In this work, we just use the physical interactions between proteins. The three other datasets are human integrated protein-protein interaction reference (HIPPIE) [21], agile protein interactomes dataanalyzer (APID) [22], and homologous interactions (Hint) [23] that contain 57,428, 171,448, and 64,399 experimental interactions, respectively. These interactions are derived from high-throughput yeast-two hybrid $(\mathrm{Y} 2 \mathrm{H})$ and mass spectrometry methods. We map all of the proteins from these five datasets to their corresponding universal protein resource (UniProt) ID [24]. We removed a protein if it could not be mapped to a Uniprot ID. Finally, in this study, we used 25,260 proteins and 304,730 interactions. For all of these proteins, we use biological process terms from gene ontology (GO) term [25] to point out the biological modules in humans. We find that 20,642 proteins from these 25,260 proteins or $81 \%$ of them are annotated. We consider a biological process annotation informative if it has these two properties. First, at least $k$ proteins are annotated with it. Second, each of its descendant's GO terms needs to have less than $k$ proteins annotated with them. We set 3 as a value of $k$ and we note that 16,021 biological processes corresponding to these 25,260 proteins that are participating in our interactions. We also use 332 human proteins involved in 26 proteins of the SARS-CoV-2 virus that were revealed by Gorden et al. [14]. The set, $T$, shows these 332 proteins as possible targets of the SARS-CoV-2 virus. For this set of 332 human proteins, we also consider 1374 IBP GO terms as high-confidence SARS-CoV-2 Human PPI.

We define the overlap between two biological processes, $p_{1}$ and $p_{2}$ in the following way $(|$.$| shows the$ size):

$$
\operatorname{Overlap}\left(p_{1}, p_{2}\right)=\frac{\left|p_{1} \bigcap p_{2}\right|^{2}}{\left|p_{1}\right|\left|p_{2}\right|} .
$$

Then, the processes with more than $15 \%$ overlaps have been removed. Through this filtering method, we have 1213 non-overlapping biological processes corresponding to COVID-19.

\section{Interactive information between drugs and human protein targets}

To evaluate our candidate targets, we use all drugs and their corresponding targets interactions that are reported in the UniProt [24]. These interactions contain 6163 drugs from All-Drug group that are reported in UniProt, these drugs have 2898 protein targets. We also use 44 experimental unapproved drugs for COVID-19 that are reported in DrugBank [26]. From these 44 drugs, 27 drugs have no target information and only 17 drugs have the drug target information. These 17 drugs can target 78 proteins in a cell. This group of drugs is denoted as Covid-Drug. The second group of drugs contains 590 drugs as clinical trials for COVID-19. From these 590 drugs, 328 drugs have targets in the PPI network denoted as Clinical-Drug. These 328 drugs can target 888 proteins in a cell.

\section{Construction of drug-target network}

We define some topological features in a PPI network for cluster identification of drugs. These features cluster the available experimental unapproved drugs for COVID-19 with respect to these topological properties of their associated targets in the PPI network. To do this, we define a drug-targets network in the following way.

Each drug-targets network is considered as a bipartite graph $H=<D, \tau, E^{*}>$. In graph $H$, nodes are divided into two different sets. The first one, $D$, demonstrates the set of experimental unapproved drugs for COVID-19, and the second one, $\tau$, shows the experimental unapproved drug targets. Each edge $e_{v d} \in E^{*}$ shows that two nodes $v \in \tau$ and $d \in D$ are connected if the node $v$ in a human cell be a target of drug $d$. In fact $\tau$, contains the proteins that are placed in the intersection of all drug targets with 2898 proteins and set $E$. Supposed that $G=<V, E>$ is a PPI network that contains the set of virus targets $(T)$ and the set of main targets $(\tau)$. Two nodes $v_{i}$ and $v_{j}$ are neighbors if there is an edge between them. Suppose that $N\left(v_{i}\right)$ shows a set of all 
neighbors for a node $v_{i}$, therefore $d\left(v_{i}\right)=\left|N\left(v_{i}\right)\right|$ indicates the degree of $v_{i}$.

We define 3 different informative topological features for each drug, $d$, and its particular targets as follows. The following features are defined with respect to the situation of its main targets and COVID-19 related biological process.

$1 D_{T}(d)$ : The average ratio of the number of neighbors for each protein $v_{i} \in \tau_{d}$ that is also placed in set $T$ according to the degree of $v_{i}$.

$$
D_{T}(d)=\frac{\sum_{i=1}^{m} \frac{\left|N\left(v_{i}\right) \cap T\right|}{d\left(v_{i}\right)}}{\left|\tau_{d}\right|},
$$

where $\tau_{d}=\left\{v_{1}, \ldots . v_{m}\right\}$ denotes the number of main targets for drug $d$.

2 The participation rate of $\tau_{d}$ in set $\pi$ defines as follow:

$$
P_{I B P}(d)=1-\sum_{p_{i} \in \pi}\left(\frac{\left|p_{i} \bigcap \tau_{d}\right|}{\sum_{p_{i} \in \pi}\left|p_{i} \bigcap \tau_{d}\right|}\right)^{2},
$$

where set $\pi=\left\{p_{1}, p_{2}, \ldots, p_{k}\right\}$ shows the non-overlapping biological processes corresponding to COVID19. The possible values for $P_{I B P}(d)$ is between 0 and 1. If the value of $P_{I B P}(d)$ is closer to 1 , it means the neighbors of node $d$ have higher distribution in the set of biological processes.

$3 D_{P}(d)$ : The average ratio of the number of neighbors for each protein $v_{i} \in \tau_{d}$ that is also placed in set $\pi$ according to the degree of $v_{i}$.

$$
D_{P}(d)=\frac{\sum_{i=1}^{m} \frac{\left|N\left(v_{i}\right) \bigcap P\right|}{d\left(v_{i}\right)}}{\left|\tau_{d}\right|} .
$$

where $P=\bigcup_{p_{i} \in \pi} p_{i}$.

\section{Clustering method based on topological features of drug targets}

Suppose that $G=<V, E, \omega>$ is a COVID-19 related biological network and $H=<\tau, D, E^{*}>$ is a bipartite drug-target graph $(\tau \subset V)$. Let $E \subset V$ be a set of essential proteins and $\tau$ be a set of main targets. Now, for each drug that has at least one target in set $\tau$, we measure the topological features $D_{P}, D_{T}$ and $P_{I B P}$ with respect to their targets in $\tau$. Suppose that $d^{*}$ is a drug from the Covid-Drug group with the corresponding values of topological features $D_{P}\left(d^{*}\right), D_{T}\left(d^{*}\right)$ and $P_{I B P}\left(d^{*}\right)$, respectively. According to the near zero threshold $\epsilon$, a drug $d$ is placed in the same cluster with $d^{*}$ if the following equation is satisfied:

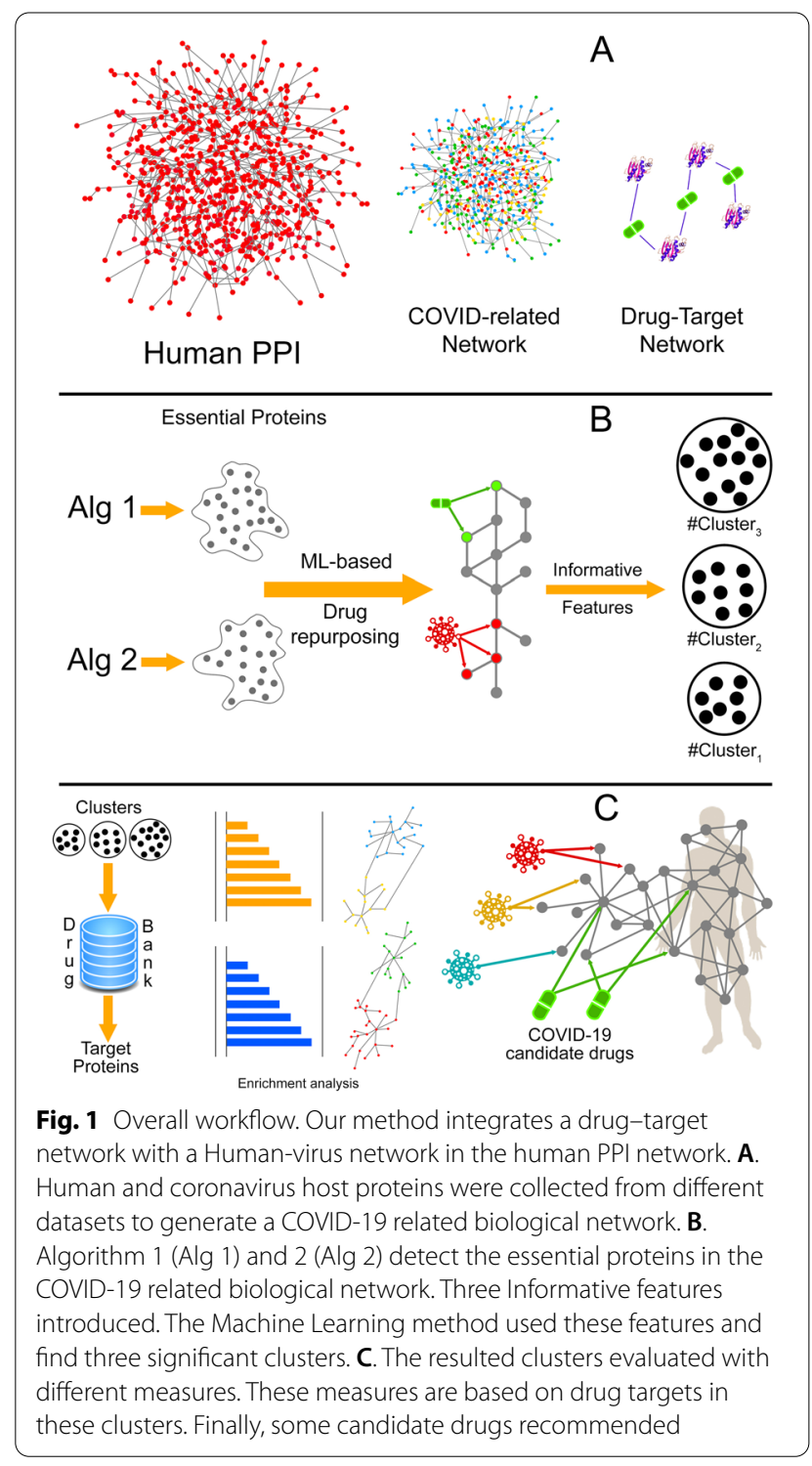

The overall view of our proposed method is illustrated in Fig. 1. Human and coronavirus host proteins were collected from different datasets to generate a COVID-19 related biological network (Part (A)). In Part B, Algorithm 1 (Alg 1) and 2 (Alg 2) are applied to detect the essential proteins in the COVID-19 related biological network. According to the defined features, the clustering method was used to find five appropriate clusters. In Part (C), the resulted clusters evaluated with different measures and some candidate drugs recommended.

$$
C=\left\{d \in D \quad \&\left|\sqrt{\left(D_{P}(d)-D_{P}\left(d^{*}\right)\right)^{2}+\left(D_{T}(d)-D_{T}\left(d^{*}\right)\right)^{2}+\left(P_{I B P}(d)-P_{I B P}\left(d^{*}\right)\right)^{2}}\right|<\epsilon\right\}
$$




\section{Results}

\section{Evaluation essential proteins related to COVID-19}

The protein sets that are resulted from algorithms 1 and 2 are placed in the sets $\mathrm{Cut}_{1}$ and $\mathrm{Cut}_{2}$, respectively. The union of $\mathrm{Cut}_{1}$ and $\mathrm{Cut}_{2}$ is placed in the Cut $t_{\text {union }}$ set and the intersection of them is placed in $\mathrm{Cut}_{\text {intersect }}$, respectively. For more evaluation of essential proteins of $\mathrm{Cut}_{1}$ and $\mathrm{Cut}_{2}$ sets, we study the topological properties of these two sets. In this work, we claim that through our cut sets as results of two presented algorithms, the maximum number of IBP GO terms are disrupted. We also claim that the two disjoint sets of vertices $G_{1}$ and $G_{2}$ (resulting from the cut set) are approximately equal in size. Moreover, each IBP GO term, like $C$ has almost the same size on both sides of $G_{1}$ and $G_{2}$ sets. Suppose that $C$ is a process from the IBP GO terms. The disruption score for this process is defined as follows [16]:

$$
\text { Score }_{\operatorname{disrupt}(C)}=\frac{\operatorname{MAX}\left\{C \cap G_{1}, C \cap G_{2}\right\}}{|C|},
$$

The closer value of Score $\operatorname{disrupt}(C)_{\text {(o) }} \frac{1}{2}$ indicates that process $C$ is completely disrupted. However, if the Score $_{\text {disrupt }(C)}$ for a process $C$ is in the range $\left[0, \frac{1}{2}+\epsilon\right]$, we say that this process is $\epsilon$-disrupted.

For more evaluation of our proposed cut sets, $\mathrm{Cut} \mathrm{t}_{1}$ and $\mathrm{Cut}_{2}$, we define two other cut sets with some important topological features. We want to compare our proposed cut sets with these two cut sets and show the advantage of our proposed cut sets with respect to the defined measure Score $\operatorname{disrupt}(C)$. In the first cut set,

Table 1 The number of $\epsilon$-disrupted processes for the selected cut sets

\begin{tabular}{lrrll}
\hline & Cut $_{\mathbf{1}}$ & Cut $_{\mathbf{2}}$ & Cut $_{\text {hub }}$ & Cut $_{\text {weight }}$ \\
\hline 0.05-disrupted & 745 & 750 & 230 & 298 \\
0.1-disrupted & 877 & 892 & 378 & 403 \\
0.2-disrupted & 1021 & 1037 & 682 & 733 \\
\hline
\end{tabular}

we select the high degree vertices such that the removal of these vertices divides the graph $G$ into two disjoint parts. This set contains hubs and we named this set as $\mathrm{Cut}_{\text {hub }}$. In the second cut set, we select the high weight vertices such that the removal of these vertices divides the graph $G$ into two disjoint parts. We named this set as $\mathrm{Cut}_{\text {weight }}$.

In Table 1 we compare the number of $\epsilon$-disrupted processes for $\mathrm{Cut}_{1}, \mathrm{Cut}_{2}, \mathrm{Cut} t_{\text {hub }}$ and $\mathrm{Cut}_{\text {weight }}$ respectively. The results of Table 1 show that $\mathrm{Cut}_{1}$ and $\mathrm{Cut}_{2}$ have better disruption properties and this confirms that the selection algorithm that we used for $\mathrm{Cut}_{1}$ and $\mathrm{Cut}_{2}$ are significantly better than other algorithms.

In Table 2, we study the number of IBP GO terms that are disrupted through these cut sets. We find that almost all sets disrupt a high number of biological processes. However, $\mathrm{Cut}_{\text {union }}$ shows significant superiority with respect to the number of drugs in Covid-Drug and Clinical-Drug groups. Table 2 shows that from these 17 drugs in the Covid-Drug group, 16 drugs are approved with $\mathrm{Cut}_{\text {union }}$, and from 328 drugs in the second group as Clinical-Drug, 273 drugs are approved

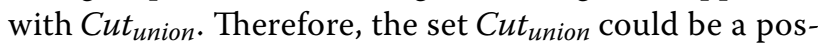
sible good candidate to find essential proteins related to COVID-19 pathology as drug targets. To find these essential proteins, we use gene-disease relationship from DAVID tools. We also study some essential proteins in $\mathrm{Cut}_{\text {union }}$ that are shared by cardiovascularrelated, hypertension, diabetes type 2 , kidney-related and lung-related diseases and placed them in a set $E$. Table 3 shows 93 proteins of set $E$ that are annotated to four out of five of these specific diseases with significant p-values. We also find that from 17 drugs in the Covid-Drug group, 10 drugs including Bevacizumab, Azithromycin, Ritonavir, Ibuprofen, Colchicine, Darunavir, Methylprednisolone, Tocilizumab, Chloroquine, and Dexamethasone. The results also show that from 328 drugs in Clinical-Drug, 185 drugs are approved by set E. Generally, among 6,163 drugs from the All-Drug

Table 2 The first row shows the number of proteins in sets T, Cut $2, \mathrm{Cut}_{1}, \mathrm{Cut}_{\text {intersect }}$ and Cut ${ }_{\text {union, }}$ respectively.

\begin{tabular}{|c|c|c|c|c|c|}
\hline & $T$ & $\mathrm{Cut}_{2}$ & Cut $_{1}$ & Cut $_{\text {intersect }}$ & $\mathrm{Cut}_{\text {union }}$ \\
\hline No. Protein & 332 & 2017 & 2100 & 1115 & 3002 \\
\hline IBP Go terms & 1374 & 1279 & 1197 & 1120 & 1306 \\
\hline No. targets in Covid-Drug & 1 & 22 & 20 & 15 & 27 \\
\hline No. targets in Clinical-Drug & 15 & 218 & 217 & 154 & 281 \\
\hline No. approved drugs in Covid-Drug & 2 & 15 & 15 & 14 & 16 \\
\hline No. approved drugs in Clinical-Drug & 30 & 246 & 260 & 225 & 273 \\
\hline
\end{tabular}

The number of IBP GO terms overlapped with these subsets collected in the second row. The number of drug targets in each drug group that are associated with these subsets are reported in the third, fourth and fifth rows, respectively. The number of drugs in each drug group that are associated with these subsets are reported in the sixth, seventh and eighth rows, respectively 
Table 3 Essential protein related to COVID-19 pathology

\section{Essential protein related to COVID-19 pathology}

O00206, O14543, O14763, O60603, P00533, P00734, P01019, P01033, P01130, P01133, P01137, P01344

P01374, P01375, P01579, P01583, P01584, P01889, P01891, P01892, P01911, P01912, P02647, P02649

P02751, P02778, P03372, P03989, P04114, P04229, P04439, P04637, P05019, P05089, P05106, P05112

P05164, P05231, P05362, P05534, P06858, P08253, P08571, P08684, P09211, P09601, P10145, P10415

P10635, P1 1021, P11226, P11473, P13498, P13500, P13501, P14210, P14780, P15692, P16035, P17813

P19438, P19838, P21549, P25445, P28482, P29279, P29459, P29474, P31645, P31749, P35222, P35354

P38936, P40763, P40933, P41597, P42336, P42345, P42898, P48023, P48061, P60568, P78423, P78527

P81172, Q04721, Q14116, Q15848, Q16236, Q30201, Q99958, Q9NR96, Q9Y2R2

group, 1689 drugs are approved by set E. These drugs target 65 proteins out of 93 proteins in set E. We also evaluate set $\mathrm{E}$ with respect to the related pathways with DAVID tools. The results show significant pathways related to COVID-19 that have been studied by previous studies [27-30]. A part of these pathways with significant $\mathrm{p}$-values are reported in Table 4.

\section{Statistical properties of clusters}

As we mentioned earlier, our clustering method introduces 5 different clusters named as \#Cluster $r_{1}$, \#Cluster 2 , \#Cluster 3 , \#Cluster ${ }_{4}$, and \#Cluster ${ }_{5}$ for 1689 drugs that are approved by proteins in set $E$. Our clustering method uses the defined topological features and 10 approved drugs in Covid-Drug that mentioned in the previous

Table 4 Some of the significantly enriched pathways that are related to COVID-19 essential proteins (E)

\begin{tabular}{|c|c|c|c|}
\hline \multirow{2}{*}{$\begin{array}{l}\text { Annotation cluster } 1 \\
\text { Term }\end{array}$} & \multicolumn{2}{|c|}{ Enrichment score: 11} & \\
\hline & Count & P value & \\
\hline hsa05142:Chagas disease (American trypanosomiasis) & 20 & $2.47 \mathrm{E}-18$ & [27] \\
\hline hsa05323:Rheumatoid arthritis & 17 & $1.79 \mathrm{E}-15$ & [27] \\
\hline hsa05144:Malaria & 14 & 4.85E-15 & [27] \\
\hline hsa05321:Inflammatory bowel disease (IBD) & 15 & $7.61 \mathrm{E}-15$ & [27] \\
\hline hsa05140:Leishmaniasis & 14 & $8.80 \mathrm{E}-13$ & [27] \\
\hline hsa05152:Tuberculosis & 19 & $9.13 \mathrm{E}-13$ & [27] \\
\hline hsa04620:Toll-like receptor signaling pathway & 15 & $1.15 \mathrm{E}-11$ & [28] \\
\hline hsa05146:Amoebiasis & 14 & $1.75 \mathrm{E}-10$ & [27] \\
\hline hsa05145:Toxoplasmosis & 14 & $2.81 \mathrm{E}-10$ & [27] \\
\hline hsa05134:Legionellosis & 10 & $8.56 \mathrm{E}-09$ & [29] \\
\hline hsa05133:Pertussis & 10 & $1.66 \mathrm{E}-07$ & [27] \\
\hline hsa04621:NOD-like receptor signaling pathway & 9 & $2.12 \mathrm{E}-07$ & [30] \\
\hline Annotation cluster 2 & \multicolumn{3}{|c|}{ Enrichment score: 8.80} \\
\hline Term & Count & P value & \\
\hline hsa05168:Herpes simplex infection & 20 & $1.31 \mathrm{E}-13$ & [29] \\
\hline hsa04940:Type I diabetes mellitus & 12 & $8.30 \mathrm{E}-13$ & {$[27]$} \\
\hline hsa05332:Graft-versus-host disease & 11 & $1.89 \mathrm{E}-12$ & \\
\hline hsa05330:Allograft rejection & 10 & $2.35 \mathrm{E}-10$ & {$[27]$} \\
\hline hsa05320:Autoimmune thyroid disease & 7 & $2.72 \mathrm{E}-05$ & [27] \\
\hline Annotation cluster 3 & \multicolumn{3}{|c|}{ Enrichment score: 4.53} \\
\hline Term & Count & P value & \\
\hline hsa04931:Insulin resistance & 10 & $3.80 \mathrm{E}-06$ & \\
\hline hsa04920:Adipocytokine signaling pathway & 8 & $1.47 \mathrm{E}-05$ & [28] \\
\hline hsa05221:Acute myeloid leukemia & 6 & $4.50 \mathrm{E}-04$ & \\
\hline
\end{tabular}


Table 5 The exceeding values (EV) for \#Cluster ${ }_{1}$,\#Cluster 2 , \#Cluster 3 ,\#Cluster 4 and \#Cluster 5

\begin{tabular}{ll}
\hline & EV values \\
\hline \#Cluster $_{1}$ & $1 \mathrm{E}-05$ \\
\#Cluster $_{2}$ & 0.00018 \\
\#Cluster $_{3}$ & 0.00109 \\
\#Cluster $_{4}$ & 0.0011 \\
\#Cluster $_{5}$ & 0.0013 \\
\hline
\end{tabular}

Table 6 The number of All-Drugs, Covid-Drug and Clinical-Drug for \#Cluster ${ }_{1}, \#$ Cluster $_{2}$, \#Cluster ${ }_{3}$, \#Cluster 4 and \#Cluster 5

\begin{tabular}{llllll}
\hline & \#Cluster $_{\mathbf{1}}$ & \#Cluster $_{2}$ & \#Cluster $_{\mathbf{3}}$ & \#Cluster $_{\mathbf{4}}$ & \#Cluster $_{\mathbf{5}}$ \\
\hline No. All-Drugs & 2 & 8 & 10 & 28 & 14 \\
No. Covid-Drug & 1 & 1 & 1 & 1 & 6 \\
No. Clinical- & 1 & 2 & 3 & 1 & 8 \\
Drug & & & & & \\
\hline
\end{tabular}

subsection, as clustering criteria. In order to evaluate \#Cluster $r_{1}$, \#Cluster 2 , \#Cluster ${ }_{3}$, \#Cluster 4 , and \#Cluster 5 , we compare our clusters with randomly generated sets. Suppose that $N_{i}=\left(a_{i}, b_{i}\right)$ for $i=1, \ldots, 10^{5}$ denotes both the number of Covid-Drug and Clinical-Drug in $i$-th randomly generated sets groups of size $n(\mathrm{n}=2,8,10,28$ and 14). In $N_{i}, a_{i}$ and $b_{i}$ denote the number of Covid-Drug and Clinical-Drug in group of size $n$, respectively. For $n$ with sizes of $2,8,10,28$ and 14, we define $\mathrm{N}=(1,1),(1,2)$ $(1,3),(1,1)$, and $(6,8)$, respectively. Let $X=\left\{i \mid N_{i}>N\right\}$ for $i=1, \ldots, 10^{5}$, where $X$ denotes the number of random sets that performed better than the proposed clusters [31]. The null hypothesis, $H_{0}$, is that our selected drug set of size $n$ is not important. The alternative hypothesis, $H_{1}$, is that our selected drug set of size $n$ is indeed important. We use exceeding value (EV) as $E V=\frac{|X|}{100000}$, where $|X|$ denotes the size of $X$. If $E V<\alpha$ then, we reject $H_{0}(\alpha$ is a threshold value that we consider to be 0.05 ). The values of EV for \#Cluster 1 , \#Cluster 2 , \#Cluster 3 , \#Cluster 4 , and \#Cluster 5 are reported in Table 5 (These values cause extremely significant results). The EV denotes the percentage of random clusters that perform better than our clusters out of $10^{5}$ random selections. We can conclude that these values are extremely significant and our selected clusters show a better performance than all of these random selections and significantly far from random selection. In Table 6 we also report some details about the proposed clusters. In this table, the number of Covid-Drug, Clinical-Drug, and drugs that are placed in All-Drug groups are reported, respectively.

\section{Evaluation of clusters with respect to proteins as drug targets}

For more evaluation, we investigate all of the proteins as drug targets in each cluster. Table 7 shows some details about our selected clusters. In this table, the first row indicates the number of proteins as drug targets in our PPI network. From these proteins, the number of important ones in each cluster that are mentioned as main targets is reported in the second row. These particular targets are the group of proteins from the first row of the table that is placed in set $\mathrm{E}$. The number of human proteins that are targeted with the virus is demonstrated as a set $T$ and reported in the third row. The fourth and fifth rows show the number of these proteins that are targeted through at least one drug in Covid-Drug and Clinical-Drug, respectively. Table 7 reports 10 drugs in \#Cluster 3 target 77 human proteins. From these 77 proteins, 10 proteins are reported as particular targets that are identified through our method. This cluster has the highest ratio of the main targets (10/77) in comparison with other clusters. Figure 2 illustrates the number of drug targets for each cluster. Drugs in each cluster can have distinctive and common targets. For example, the union of all targets for two drugs in \#Cluster 1 contains 28 proteins. From these 28 proteins, 17 of them are common between these two drugs. From these 17 proteins, 1 of them is placed in set $E$ that is mentioned as main target.

Table 7 The first row indicates the number of proteins as drug targets in our PPI network

\begin{tabular}{|c|c|c|c|c|c|}
\hline & \#Cluster $_{1}$ & \#Cluster 2 & $\#$ Cluster $_{3}$ & $\#$ Cluster $_{4}$ & \#Cluster 5 \\
\hline No. targets & 28 & 37 & 77 & 155 & 118 \\
\hline No. main targets & 2 & 2 & 10 & 10 & 14 \\
\hline No. targets in $T$ & 1 & 1 & 1 & 3 & 0 \\
\hline No. targets in Covid-Drug & 23 & 18 & 29 & 43 & 47 \\
\hline No. targets in Clinical-Drug & 28 & 32 & 69 & 140 & 107 \\
\hline
\end{tabular}

From these proteins, the number of important ones in each cluster is reported in the second row. The number of human proteins that are targeted with the virus is reported in the third row. The fourth and fifth rows show the number of these proteins that are targeted through at least one drug in Covid-Drug and Clinical-Drug, respectively 


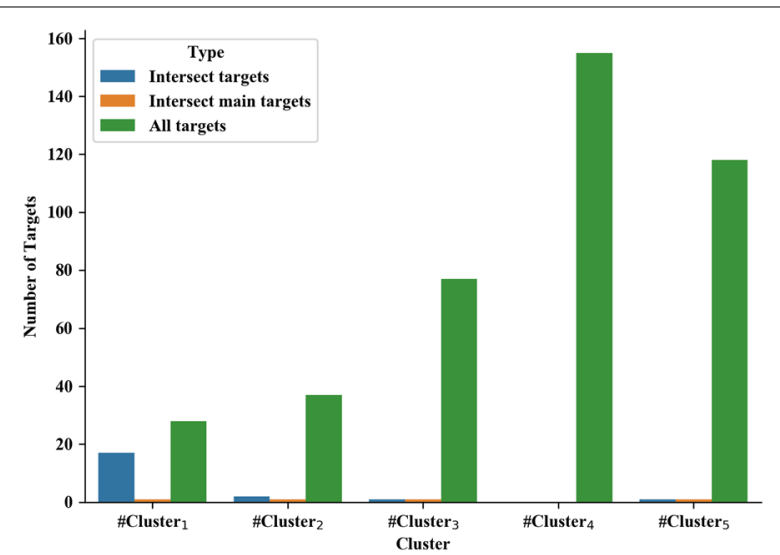

Fig. 2 The blue columns show the common drug targets for each cluster. The orange columns show the number of main targets that are common between drugs in each cluster. The green columns show all of the targets for drugs in each cluster

Figure 2 also shows the number of main targets in each cluster. Each drug in these five clusters has one or multiple main targets. From these main targets three of them are common among all drugs of each clusters. These three main targets are Vascular Endothelial Growth Factor (VEGF)-A, Cytochrome P450 3A4 (CYP3A4), and Prostaglandin-endoperoxide synthase 2 (PTGS2) or Cyclo-oxygenase2 (COX-2), respectively. Despite the lack of evidence for COVID-19, previous research shows that the VEGF family (VEGFs) has a connection with COVID19. A recent study shows that VEGFs are involved in "cytokine storm" inflammatory response. They claim that these genes may be used as prospective biomarkers for early diagnosis in COVID-19 patients [32]. The VEGFs can also be used for targeted drug delivery in COVID-19 treatment.

The second main target is PTGS2 or COX-2, which has been the subject of many studies on its association with COVID-19 and is a pro-inflammatory enzyme. Some studies showed that the structural proteins of the SARS$\mathrm{CoV}$ family are reported to influence the expression of COX-2 and the increased expression of plasma PGE2 in the blood of SARS-CoV-infected patients. It is also reported that COX-2 plays a crucial role in limiting the anti-viral cytokine response to viral infection. Therefore, the use of an effective COX-2 inhibitor during early viral infection could enhance interferon responses. It might also increase anti-viral immunity [33]. The result of [34] study shows the importance of VEGF-A and COX-2 in relation to COVID-19. In this study, PPI analysis was used to find the hub genes linked to COVID-19 and lung cancer. Among the suggested hub genes, VEGF-A and COX-2 have been confirmed and could be used as biomarkers for COVID-19.

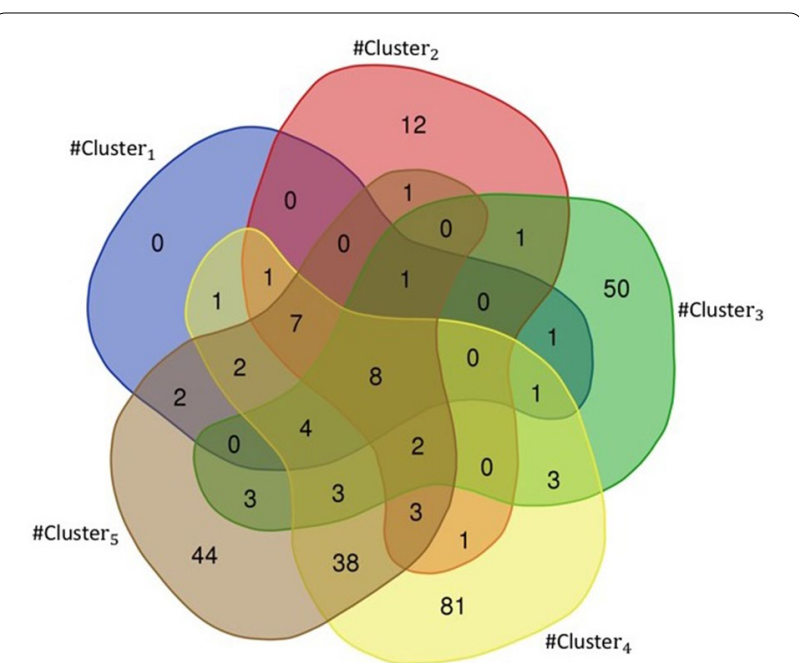

Fig. 3 The Venn diagram shows the relation of targets for \#Cluster ${ }_{1}$, \#Cluster 2 , \#Cluster 3 , \#Cluster 4 and \#Cluster 5

The next main target is Cytochrome P450 3A4 (CYP3A4). Cytochromes P450 (CYPs) is a superfamily of metabolizing enzymes. The CYP enzymes can be suppressed by an infection-related cytokine increase and inflammation. A recent study demonstrated that, like other viral infections, during the progression of COVID-19 local and systemic inflammation as well as the "cytokine storm" will potentially cause downregulation of the major CYP enzymes including CYP3A4 [35]. A new study proposed that COVID-19 pharmacogenetic studies include CYP3A4 variants [36]. The [33] study shows that CYPs metabolic activity will be surely changed during the SARS-CoV-2 infection in a similar manner, resulting in a pharmacokinetic interaction with the recommended drugs for COVID-19 treatments. In addition, liver involvement in COVID-19 may further complicate this problem, especially for drugs like remdesivir and chloroquine as COVID-19 treatments. Since remdesivir undergoes extensive metabolism by CYPs and chloroquine is also hepatically metabolized, understanding the nature of these drug-disease interactions is highly essential and can affect the therapeutic response of COVID-19 patients.

The Venn diagram in Fig. 3 illustrates the relationship between targets in 5 clusters. Our results show that despite the fact that the drugs in these five clusters are different from each other but they have 8 specific proteins as targets that are jointly targeted by the drugs in all five clusters. In addition, Fig. 3 shows that $64 \%$ (50/77) of the proteins as drug targets in \#Cluster 3 are different from the targets in other clusters, and all proteins as drug targets in \#Cluster ${ }_{1}$ are targeted by at least one drug in the other clusters. In Fig. 4, as an example, 
we show that the common targets and the total number of targets that are selected through our method with respect to the defined topological features for \#Cluster ${ }_{1}$.

\section{Evaluation of clusters with respect to related diseases}

We have studied the diseases associated with each of the drugs in each cluster based on the information on the Drugbank website. The Venn diagram in Fig. 5 shows the relationship between diseases that are associated with drug targets in each cluster. Figure 5 shows that there is no specific disease that is associated with all clusters. Respiratory Tract Infections and Type 2 Diabetes are two of six diseases that have common targets in\#Cluster 2 and \#Cluster 3 . Diabetic Macular Edema (DME) is one of two diseases that have common targets in \#Cluster 3 and \#Cluster ${ }_{5}$. Rheumatoid Arthritis and Ankylosing Spondylitis (AS) are two of twelve diseases that have common targets in \#Cluster ${ }_{4}$ and \#Cluster ${ }_{5}$.

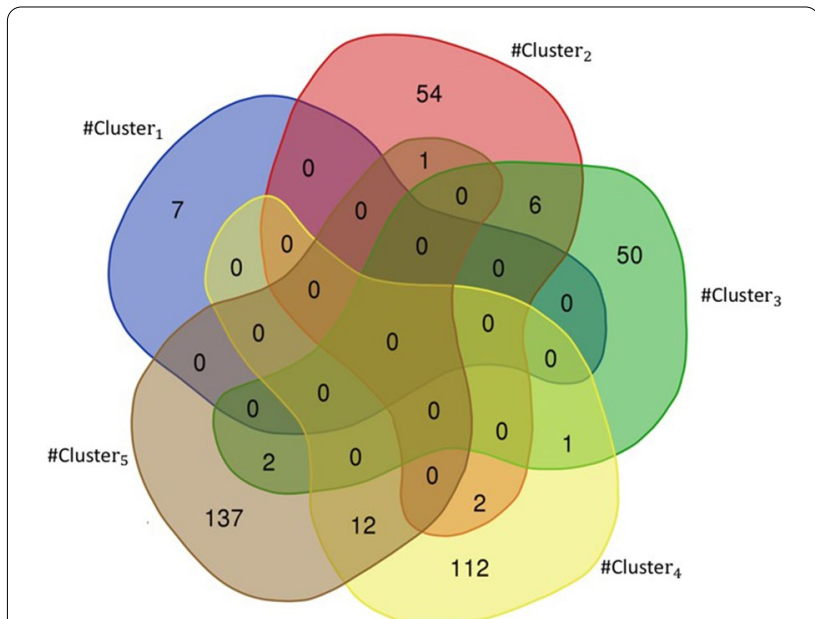

Fig. 5 The relationship between diseases that are associated with drug targets in \#Cluster ${ }_{1}$ \#Cluster ${ }_{2}$, \#Cluster ${ }_{3}$, \#Cluster 4 and \#Cluster 5

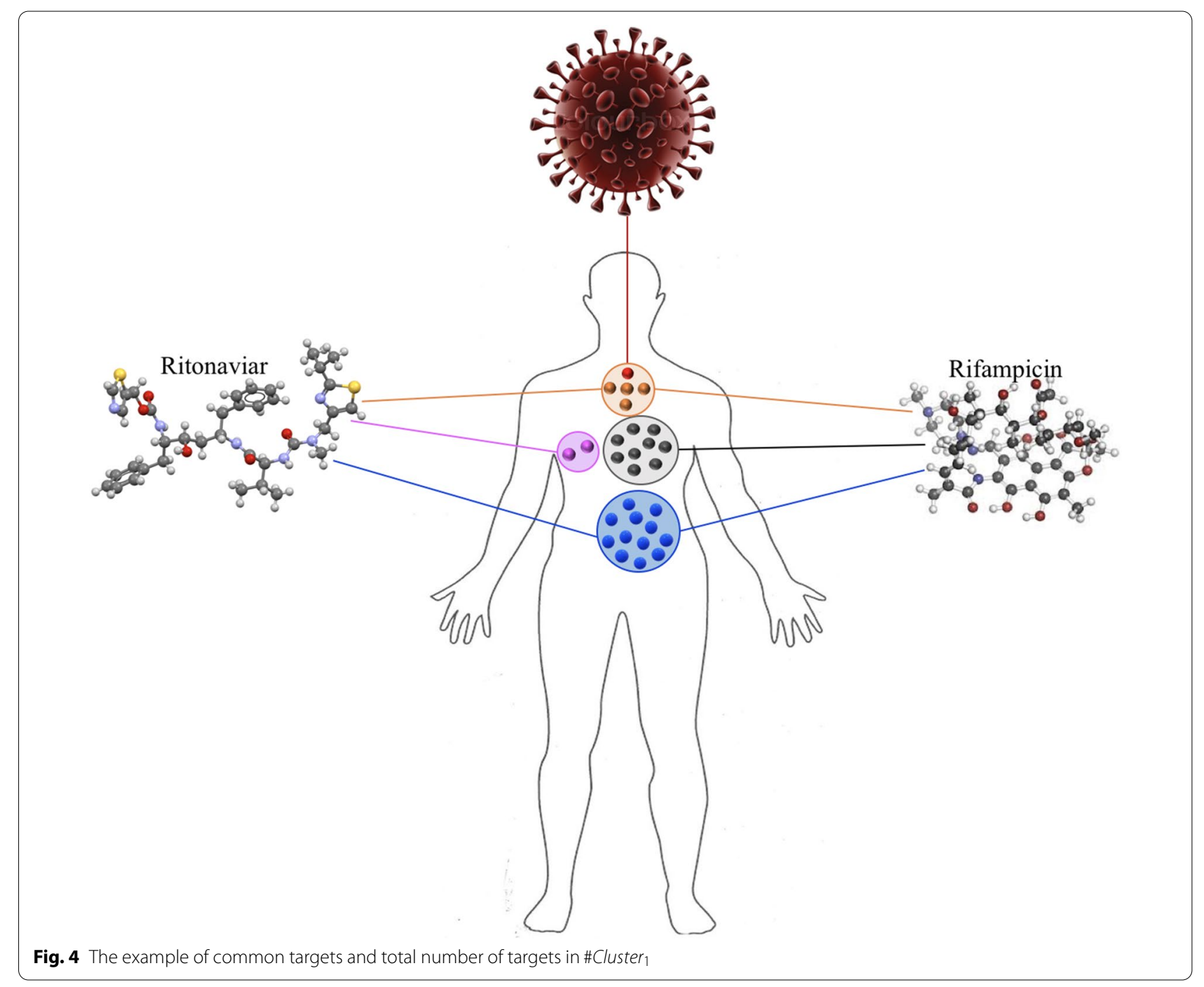


Table 8 List of the repositioning candidates, therapeutic category and the supporting published evidence in \#Cluster 1

\begin{tabular}{lll}
\hline Candidate drug & Therapeutic category & References \\
\hline Ritonavir & $\begin{array}{l}\text { Anti-HIV agents and anti-infective } \\
\text { agents }\end{array}$ & {$[26]$} \\
Rifampicin & $\begin{array}{l}\text { Anti-bacterial agents and anti-infective } \\
\text { agents }\end{array}$ & {$[38]$} \\
& [3]
\end{tabular}

The drug in Clinical-Drug group is highlighted in italic

Table 9 List of the repositioning candidates, therapeutic category and the supporting published evidence in \#Cluster 2

\begin{tabular}{lll}
\hline Candidate drug & Therapeutic category & References \\
\hline Mycophenolate mofetil & Anti-bacterial and anti-infective & {$[39]$} \\
Erythromycin & Anti-bacterial and anti-infective & {$[40]$} \\
Azithromycin & Anti-bacterial and anti-infective & {$[26]$} \\
Cerivastatin & Anticholesteremic agents & {$[41]$} \\
Ezetimibe & Anticholesteremic agents & {$[42]$} \\
Fusidic acid & Anti-bacterial and anti-infective & - \\
Canagliflozin & Alimentary tract and metabolism & {$[43]$} \\
Letermovir & Antiviral agents & {$[44]$}
\end{tabular}

Drugs in Clinical-Drug group are highlighted in italic

Table 10 List of the repositioning candidates, therapeutic category and the supporting published evidence in \#Cluster 3

\begin{tabular}{lll}
\hline Candidate drug & Therapeutic category & References \\
\hline Bevacizumab & $\begin{array}{l}\text { Angiogenesis inhibitors and } \\
\text { antibodies }\end{array}$ & {$[26]$} \\
Minocycline & $\begin{array}{l}\text { Anti-bacterial and anti-infective } \\
\text { agents }\end{array}$ & {$[45]$} \\
Gliclazide & Alimentary tract and metabolism & {$[46]$} \\
Carvedilol & Adrenergic agents & {$[47]$} \\
Ranibizumab & Angiogenesis inhibitors and & - \\
& antibodies & \\
Tromethamine & Drug delivery systems & {$[48]$} \\
Vandetanib & Antineoplastic agents & - \\
Veglin & - & - \\
Denibulin & Heterocyclic compounds & {$[49]$} \\
Foreskin keratinocyte & Allogeneic cultured cell scaffold & {$[50]$} \\
\hline
\end{tabular}

Drugs in Clinical-Drug group are highlighted in italic

\section{Drug repurposing candidate}

In this section, the drug clusters identified with our proposed method have been analyzed to infer some useful drug repurposing candidates. Tables 8, 9, 10, 11 and 12 show repurposing candidates in \#Cluster 1 , \#Cluster 2 , \#Cluster 3 , \#Cluster 4 , and \#Cluster ${ }_{5}$, contain 2, 8, 10, 28, and 14 drugs, respectively. Our clustering method enables us to partition the large drug-related network into
Table 11 List of the repositioning candidates, therapeutic category and the supporting published evidence in \#Cluster 4

\begin{tabular}{|c|c|c|}
\hline Candidate drug & Therapeutic category & References \\
\hline Aldesleukin & Anti-infective agents & {$[26]$} \\
\hline Dapsone & Anti-infective agents & {$[51]$} \\
\hline Acetaminophen & Central nervous system agents & {$[52]$} \\
\hline Celecoxib & Anti-inflammatory agents & {$[33]$} \\
\hline Rofecoxib & Anti-inflammatory agents & {$[53]$} \\
\hline Valdecoxib & Anti-inflammatory agents & {$[54]$} \\
\hline Diclofenac & Anti-inflammatory agents & {$[55]$} \\
\hline Triamcinolone & Alimentary tract and metabolism & - \\
\hline Etoposide & Antineoplastic agents & {$[56]$} \\
\hline Phenylbutazone & Anti-inflammatory agents & {$[57]$} \\
\hline Meloxicam & Anti-inflammatory agents & {$[58]$} \\
\hline Chlorphenesin & Central nervous system agents & {$[59]$} \\
\hline Ibuprofen & Anti-inflammatory agents & {$[26]$} \\
\hline Paclitaxel & Antineoplastic agents & - \\
\hline Drospirenone & Contraceptive agents, female & {$[60]$} \\
\hline Antipyrine & Anti-inflammatory agents & - \\
\hline Etoricoxib & Anti-inflammatory agents & [53] \\
\hline Resveratrol & Anti-inflammatory agents & {$[61]$} \\
\hline Nimesulide & Anti-inflammatory agents & {$[62]$} \\
\hline Capsaicin & Sensory system agents & {$[63]$} \\
\hline Parecoxib & Anti-inflammatory agents & {$[64]$} \\
\hline Pomalidomide & Antineoplastic agents & {$[65]$} \\
\hline Cannabidiol & Antidepressive agents & {$[66]$} \\
\hline Loxoprofen & Anti-inflammatory agents & {$[67]$} \\
\hline Dexibuprofen & Anti-inflammatory agents & {$[68]$} \\
\hline Propacetamol & Sensory system agents & {$[69]$} \\
\hline Venetoclax & Antineoplastic agents & - \\
\hline Nabiximols & Central nervous system agents & - \\
\hline
\end{tabular}

Drugs in Clinical-Drug group are highlighted in italic

Table 12 List of the repositioning candidates, therapeutic category and the supporting published evidence in \#Cluster 5

\begin{tabular}{lll}
\hline Candidate drug & Therapeutic category & References \\
\hline Colchicine & Immunosuppressive agents & {$[26]$} \\
Darunavir & Anti-infective agents & {$[26]$} \\
Dexamethasone & Anti-inflammatory agents & {$[26]$} \\
Methylprednisolone & Anti-inflammatory agents & {$[26]$} \\
Tocilizumab & Antirheumatic agents & {$[26]$} \\
Acetaminophen & Central nervous system agents & - \\
Chloroquine & Anti-inflammatory agents & {$[26]$} \\
Donepezil & Central nervous system agents & {$[70]$} \\
Clomipramine & Central nervous system agents & {$[71]$} \\
Resveratrol & Anti-inflammatory agents & {$[72]$} \\
Curcumin & Anti-inflammatory agents & {$[73]$} \\
Curcumin sulfate & anti-inflammatory agent & {$[74]$} \\
Baricitinib & Antineoplastic and immunomod- & {$[37]$} \\
& ulating agents & \\
Lidocaine & Antiarrhythmic agents & {$[75]$} \\
\hline
\end{tabular}

Drugs in Clinical-Drug group are highlighted in italic 
smaller subgroups and this can simplify the drug repurposing process. Tables $8,9,10,11$ and 12 show the mechanism of action, therapeutic category, and supporting published evidence for each drug in \#Cluster ${ }_{1}$, \#Cluster ${ }_{2}$, \#Cluster $r_{3}$, \#Cluster 4 , and \#Cluster 5 , respectively. From these 62 drugs, 10 of them have previously been proposed as potential drug repurposing candidates for COVID-19 disease.

\section{Discussion and summary}

Researchers have been searching for efficient medications to prevent or cure COVID-19 since the first case was discovered in 2019. To advance this goal, we introduced the four steps method. In the first step, the COVID19 related biological network was constructed and the essential proteins that have a wide range of important functions in the biological network were detected. In the second step, we focused on finding the most effective essential proteins related to COVID-19. To do this, we used two different algorithms to identify the minimum number of proteins that participate in a large number of IBP GO terms and placed them in two distinct sets. Then, we evaluated proteins of these two sets with respect to the number of approved Covid-Drug and Clinical-Drug by them (Table 2). We placed the union of these two sets in the set Cut $_{\text {union }}$ and studied set Cut union with respect to the number of IBP GO terms that are disrupted. As a result, the selected proteins can be identified as a suitable candidate set for the COVID-19. It is noticeable that not every essential protein is an appropriate candidate as an essential protein for COVID-19 pathology. Some of these essential proteins are related to the cellular function of the cell and selecting them as drug targets may lead to disruption of cellular function. Considering that, in the third step, we picked candidate proteins directly related to COVID-19 pathology. For the final essential protein selection process in this step, we identified proteins that were associated with underlying diseases such as cardiovascular disease, diabetes, hepatitis, lung, kidney diseases, and various types of cancer. Among 3,002 essential proteins related to COVID-19 in $\mathrm{Cut}_{\text {union }}$, we detected 93 proteins associated with at least four of five underlying mentioned diseases as essential proteins related to COVID-19 pathology (Table 3). We evaluated these proteins with respect to the related pathways with DAVID tools (Table 4). As a result, these selected proteins could be suitable candidates as drug targets for COVID-19 treatment. In the fourth step, multiple informative topological features for drug-target and a PPI network were proposed. Our methods tried to find significant clusters containing appropriate candidate drugs through these features. These features cluster the available experimental unapproved drugs for COVID-19 into five groups (\#Cluster 1 , \#Cluster 2 , \#Cluster ${ }_{3}$, \#Cluster 4 , and \#Cluster $\left.{ }_{5}\right)$. These clusters have a significant difference from random clusters (Table 5) and contain a significant number of Covid-Drug and Clinical-Drug (Table 6). We also used three different measures for validating the obtained clusters. The first measure was based on the proteins as drug targets in these clusters, we showed that the proposed clusters have meaningful targets that were known in recent studies as COVID-19 targets (Table 6 and Figs. 2, 3, and 4). The second measure was based on the related diseases that have drugs in our clusters. We found some related diseases like DME and Rheumatoid Arthritis that have drugs in two of our clusters (Fig. 5). The third measure was related to drugs as good candidates for drug repurposing in COVID-19 treatment.

In summary, the main advantage of our method in comparison to other studies was clustering FDAapproved drugs that are related to COVID-19 according to the biological and topological properties of their targets. It can be concluded that partitioning the drugrelated network into smaller networks (clusters) can improve drug repurposing results for clinical trials. In this work, we proposed some good drug candidates as repurposing candidates for COVID-19 treatment. Our results showed that most of our drug candidates were used in clinical trials or suggested in at least one study as suitable drug repurposing candidates (Tables 7-11). Our results also revealed that the proposed informative features recommended some suitable candidate drugs like [37] and Rifampicin [38]. Finally, this study offered powerful network-based informative features for the fast identification of repurposable drugs as a potential treatment for COVID-19. The proposed method can effectively minimize the timing gap between preclinical testing conclusions and clinical results, which is a considerable problem in the fast development of efficient treatment strategies for the emerging COVID-19 outbreak.

\section{Abbreviations \\ COVID-19: The coronavirus disease 2019; PPI: Protein-protein interaction; IBP: Informative biological processes; DAVID: Database for annotation, visualization, and integrated discovery; BioGRID: Biological general repository for interaction datasets; HIPPIE: Human integrated protein-protein inter-action reference; APID: Agile protein interactomes dataanalyzer; Hint: Homologous interactions; Y2H: yeast-two hybrid; UniProt: Universal protein resource; GO: Gene ontology; EV: Exceeding value; CYP3A4: Cytochrome P450 3A4; VEGF: Vascular endothe- lial growth factor; CYP3A4: P450 3A4; PTGS2: Prostaglandin-endoperoxide synthase 2; COX-2: Cyclo-oxygenase2; CYPs: Cytochromes P450.}

\section{Acknowledgements}

None.

Authors' contributions

M. H and G. T developed the methods. M. H, G. T and R. A performed computational and statistical analysis. M. H, G. T and R. A design and wrote the paper. G. T and R. A contributed in writing and editing the manuscript. The authors 
contributed equally to this work. All authors read and approved the final manuscript.

\section{Funding}

No funding to declare.

\section{Availability of data and materials}

Datasets and the codes of the algorithms are available in our github repository, [https://github.com/rosaaghdam/Drug-Target.]

\section{Declarations}

\section{Ethics approval and consent to participate \\ None.}

\section{Competing interests}

The authors declare that they have no competing interests.

\section{Author details}

${ }^{1}$ School of Biological Sciences, Institute for Research in Fundamental Sciences (IPM), Tehran, Iran. ${ }^{2}$ Department of Mathematics, Qazvin Branch, Islamic Azad University, Qazvin, Iran. ${ }^{3}$ Department of Electrical Engineering and Computer Science, KTH Royal Institute of Technology, Stockholm, Sweden. ${ }^{4}$ Science for Life Laboratory, Stockholm, Sweden.

Received: 24 March 2021 Accepted: 6 September 2021 Published online: 20 September 2021

\section{References}

1. Smith T, Bushek J, LeClaire A, Prosser T (2020) COVID-19 drug therapy. Elsevier 1(1):1-25

2. Li J, Zheng S, Chen B, Butte AJ, Swamidass SJ, Lu Z (2016) A survey of current trends in computational drug repositioning. Brief Bioinform 17(1):2-12

3. Habibi M, Taheri G (2021) Topological network based drug repurposing for coronavirus 2019. PLoS One 16(7):0255270

4. Bolgár B, Arany A, Temesi G, Balogh B, Antal P, Matyus P (2013) Drug repositioning for treatment of movement disorders: from serendipity to rational discovery strategies. Curr Top Med Chem 13(18):2337-2363

5. Forst CV (2010) Host-pathogen systems biology. In: Infectious disease informatics, pp. 123-147

6. Kaufmann SH, Dorhoi A, Hotchkiss RS, Bartenschlager R (2018) Hostdirected therapies for bacterial and viral infections. Nat Rev Drug Discov 17(1):35

7. Cheng F, Lu W, Liu C, Fang J, Hou Y, Handy DE, Wang R, Zhao Y, Yang Y, Huang J et al (2019) A genome-wide positioning systems network algorithm for in silico drug repurposing. Nat Commun 10(1):1-14

8. Alaimo S, Pulvirenti A (2019) Network-based drug repositioning: approaches, resources, and research directions. In: Vanhaelen Q (ed) Computational methods for drug repurposing, vol 1903. Humana Press, New York, pp 97-113

9. Bandyopadhyay S, Ray S, Mukhopadhyay A, Maulik U (2015) A review of in silico approaches for analysis and prediction of HIV-1-human proteinprotein interactions. Brief Bioinform 16(5):830-851

10. Mukhopadhyay A, Maulik U (2014) Network-based study reveals potential infection pathways of hepatitis-c leading to various diseases. PLoS One 9(4):94029

11. Cao H, Zhang Y, Zhao J, Zhu L, Wang Y, Li J, Feng Y-M, Zhang N (2017) Prediction of the Ebola virus infection related human genes using protein-protein interaction network. Comb Chem High Throughput Screen 20(7):638-646

12. Zhou Y, Hou Y, Shen J, Huang Y, Martin W, Cheng F (2020) Network-based drug repurposing for novel coronavirus 2019-NCOV/SARS-COV-2. Cell Discov 6(1):1-18

13. Li X, Yu J, Zhang Z, Ren J, Peluffo AE, Zhang W et al (2021) Network bioinformatics analysis provides insight into drug repurposing for COVID-19. Med Drug Discov 10(1):100090
14. Gordon DE, Jang GM, Bouhaddou M, Xu J, Obernier K, White KM, O'Meara MJ, Rezelj VV, Guo JZ, Swaney DL et al (2020) A SARS-COV-2 protein interaction map reveals targets for drug repurposing. Nature 583(1):1-13

15. Dick K, Biggar KK, Green JR (2020) Computational prediction of the comprehensive SARS-CoV-2 vs. human Interactome to guide the design of therapeutics. bioRxiv. https://doi.org/10.1101/2020.03.29.014381

16. Taheri G, Habibi M, Wong L, Eslahchi C (2013) Disruption of protein complexes. J Bioinform Comput Biol 11(3):1341008

17. Habibi M, Khosravi P (2018) Disruption of the protein complexes from weighted complex networks. IEEE/ACM Trans Comput Biol Bioinform 17(1):102-109

18. Taheri G, Khonsari A, Entezari-Maleki R, Sousa L (2020) A hybrid algorithm for task scheduling on heterogeneous multiprocessor embedded systems. Appl Soft Comput 91(1):106202

19. Luck K, Kim D-K, Lambourne L, Spirohn K, Begg BE, Bian W, Brignall R, Cafarelli T, Campos-Laborie FJ, Charloteaux B et al (2020) A reference map of the human binary protein interactome. Nature 580(7803):402-408

20. Chatr-Aryamontri A, Oughtred R, Boucher L, Rust J, Chang C, Kolas NK, O'Donnell L, Oster S, Theesfeld C, Sellam A et al (2017) The biogrid interaction database: 2017 update. Nucleic Acids Res 45(D1):369-379

21. Alanis-Lobato G, Andrade-Navarro MA, Schaefer MH (2016) Hippie v2. 0: enhancing meaningfulness and reliability of protein-protein interaction networks. Nucleic Acids Res 45(D1):408-414

22. Alonso-López D, Campos-Laborie FJ, Gutiérrez MA, Lambourne L, Calderwood MA, Vidal M, De Las Rivas J (2019) Apid database: redefining protein-protein interaction experimental evidences and binary interactomes. Database 2019(D1):1-10

23. Patil A, Nakamura H (2005) Hint: a database of annotated protein-protein interactions and their homologs. Biophysics 1(1):21-24

24. Consortium U (2019) Uniprot: a worldwide hub of protein knowledge. Nucleic Acids Res 47(1):506-515

25. Ontology $G$ (2019) The gene ontology resource: 20 years and still going strong. Nucleic Acids Res 47(1):330-338

26. Wishart DS, Feunang YD, Guo AC, Lo EJ, Marcu A, Grant JR, Sajed T, Johnson D, Li C, Sayeeda Z et al (2018) Drugbank 5.0: a major update to the drugbank database for 2018. Nucleic Acids Res 46(D1):1074-1082

27. Kim M, Kim YB (2020) In silico synergistic drug repurposing for combating novel coronavirus (COVID-19) outbreaks. Res Sq. https://doi.org/10. 21203/rs.3.rs-21849/v1

28. Oh KK, Adnan M, Cho DH (2020) SARS-COV-2 intervened by nsaids: a network pharmacology approach to decipher signaling pathway and interactive genes. Res Sq. https://doi.org/10.21203/rs.3.rs-111615/v1

29. Dai W, Cao D, Zhang W, Wei Y, Ding D, Li B, Gao Y, Zhao L, Jiang Y, Kong X (2020) Integrated bioinformatics analysis reveals key candidate genes and cytokine pathways involved in COVID-19 after rhinovirus infection in asthma patients. Med Sci Monit 26:e928861-1

30. Toraih EA, Sedhom JA, Dokunmu TM, Hussein MH, Ruiz EM, Muthusamy $K$, Zerfaoui M, Kandil E (2021) Hidden in plain sight: the effects of BCG vaccination in the COVID-19 pandemic. J Med Virol 93(4):1950-1966

31. Aghdam R, Ganjali M, Zhang X, Eslahchi C (2015) Cn: a consensus algorithm for inferring gene regulatory networks using the sorder algorithm and conditional mutual information test. Mol BioSyst 11(3):942-949

32. Veerabathiran R, Ragunath B, Kaviarasan V, Mohammed V, Ahmed SS (2021) Identification of selected genes associated with the SARS-COV-2: a therapeutic approach and disease severity. Bull Natl Res Centre 45(1):1-11

33. Baghaki S, Yalcin CE, Baghaki HS, Aydin SY, Daghan B, Yavuz E (2020) Cox2 inhibition in the treatment of COVID-19: Review of literature to propose celecoxib repositioning for randomized controlled studies. Int J Infect Dis 101(1):29-32

34. Nana KS, Karuppanan K, Kumar S (2021) Identification of common key genes and pathways between covid-19 and lung cancer by using protein-protein interaction network analysis. bioRxiv. https://doi.org/10. 1101/2021.02.16.431364

35. El-Ghiaty MA, Shoieb SM, El-Kadi AO (2020) Cytochrome p450-mediated drug interactions in COVID-19 patients: current findings and possible mechanisms. Med Hypotheses 144:1 10033

36. Fricke-Galindo I, Falfán-Valencia R (2021) Pharmacogenetics approach for the improvement of COVID-19 treatment. Viruses 13(3):413

37. Kalil AC, Patterson TF, Mehta AK, Tomashek KM, Wolfe CR, Ghazaryan V, Marconi VC, Ruiz-Palacios GM, Hsieh L, Kline S et al (2020) Baricitinib 
plus remdesivir for hospitalized adults with COVID-19. N Engl J Med 384(9):1-13

38. Tripathi V, Pathak Y, Mishra A (2020) Rifampicin may be repurposed for COVID-19 treatment: Insights from an in-silico study. Res Sq. https://doi. org/10.21203/rs.3.rs-22546/v1

39. Russell B, Moss C, George G, Santaolalla A, Cope A, Papa S, Van Hemelrijck M (2020) Associations between immune-suppressive and stimulating drugs and novel COVID-19-a systematic review of current evidence. Ecancermedicalscience 14(1022):1-17

40. Habibi M, Taheri G, Aghdam R (2021) A SARS-COV-2 (COVID-19) biological network to find targets for drug repurposing. Sci Rep 11(1):1-15

41. Rodrigues-Diez RR, Tejera-Muñoz A, Marquez-Exposito L, Rayego-Mateos S, Santos Sanchez L, Marchant V, Tejedor Santamaria L, Ramos AM, Ortiz A, Egido J et al (2020) Statins: could an old friend help in the fight against COVID-19. Br J Pharm 177(21):4873-4886

42. Israel A, Schäffer AA, Cicurel A, Feldhamer I, Tal A, Cheng K, Sinha S, Schiff E, Lavie G, Ruppin E (2020) Large population study identifies drugs associated with reduced COVID-19 severity. medRxiv. https://doi.org/10.1101/ 2020.10.13.20211953

43. Kouznetsova VL, Zhang A, Tatineni M, Miller MA, Tsigelny IF (2020) Potential COVID-19 papain-like protease PLPRO inhibitors: repurposing FDA-approved drugs. PeerJ 8(1):9965

44. Cantürk S, Singh A, St-Amant P, Behrmann J (2020) Machine-learning driven drug repurposing for COVID-19. arXiv preprint arXiv:2006.14707

45. Singh H, Kakkar AK, Chauhan P (2020) Repurposing minocycline for COVID-19 management: mechanisms, opportunities, and challenges. Expert Rev Anti-infect Therapy 18(10):997-1003

46. Tomar PPS, Arkin IT (2020) SARS-COV-2 e protein is a potential ion channel that can be inhibited by gliclazide and memantine. Biochem Biophys Res Commun 530(1):10-14

47. Skayem C, Ayoub N (2020) Carvedilol and COVID-19: a potential role in reducing infectivity and infection severity of SARS-COV-2. Am J Med Sci 360(3):300

48. Karimi-Zarchi M, Neamatzadeh H, Dastgheib SA, Abbasi H, Mirjalili SR, Behforouz A, Ferdosian F, Bahrami R (2020) Vertical transmission of coronavirus disease 19 (COVID-19) from infected pregnant mothers to neonates: a review. Fetal Pediatr Pathol 39(3):246-250

49. Cavasotto CN, Di Filippo Jl (2020) In silico drug repurposing for COVID-19: targeting SARS-COV-2 proteins through docking and quantum mechanical scoring. Res Sq. https://doi.org/10.26434/chemrxiv.12110199.v1

50. Rivero I, Castresana M, Guala D, Sonnhammer E (2020) Drug repurposing improves disease targeting 11 -fold on average. New insights from network module targeting and its application to COVID-19. https://doi. org/10.31219/osf.io/4rvaz

51. Farouk A, Salman S (2020) Dapsone and doxycycline could be potential treatment modalities for COVID-19. Med Hypotheses 140(1):109768

52. Rodríguez-Morales AJ, Cardona-Ospina JA, Murillo-Muñoz MM (2020) Gastroenterologists, hepatologists, COVID-19 and the use of acetaminophen. Clin Gastroenterol Hepatol 18(9):2142-2143

53. Organization WH (2020) The use of non-steroidal anti-inflammatory drugs (nsaids) in patients with COVID-19: scientific brief, 19 April 2020. Technical documents

54. Dayer MR (2020) Old drugs for jak-stat pathway inhibition in COVID-19. arXiv preprint arXiv:2010.12332

55. Kelleni MT (2020) Early use of non-steroidal anti-inflammatory drugs in COVID-19 might reverse pathogenesis, prevent complications and improve clinical outcomes. Biomed Pharmacother 133(1):110982

56. Takami A (2020) Possible role of low-dose etoposide therapy for hemophagocytic lymphohistiocytosis by COVID-19. Int J Hematol 112(1):122-124

57. Loganathan T, Ramachandran S, Shankaran P, Nagarajan D et al (2020) Host transcriptome-guided drug repurposing for COVID-19 treatment: a meta-analysis based approach. PeerJ 8(1):9357
58. Farag A, Wang P, Ahmed M, Sadek H (2020) Identification of FDA approved drugs targeting COVID-19 virus by structure-based drug repositioning. ChemRxiv. https://doi.org/10.26434/chemrxiv.12003930.v1

59. Mummed Y (2020) Molecular targets for COVID-19 drug development: enlightening nigerians about the pandemic and future treatment. Biosaf Health 2(4)

60. Regidor P, Colli E (2021) SARS COVID-19 as an immunothrombotic disease and the potential benefits of a new estrogen-free contraceptive containing drospirenone. Thromb Haemost Res 5(1):1052

61. Giordo R, Zinellu A, Eid AH, Pintus G (2021) Therapeutic potential of resveratrol in COVID-19-associated hemostatic disorders. Molecules 26(4):856

62. Scalise M, Indiveri C (2020) Repurposing nimesulide, a potent inhibitor of the b0at1 subunit of the SARS-COV-2 receptor, as a therapeutic adjuvant of COVID-19. SLAS DISCOVERY Adv Sci Drug Discov 25(10):1171-1173

63. Gonzalez-Paz LA, Lossada CA, Moncayo LS, Romero F, Paz JL, VeraVillalobos J, Pérez AE, San-Blas E, Alvarado YJ (2020) Theoretical molecular docking study of the structural disruption of the viral $3 \mathrm{cl}$-protease of COVID19 induced by binding of capsaicin, piperine and curcumin part 1: a comparative study with chloroquine and hydrochloroquine two antimalaric drugs. Res Sq. https://doi.org/10.21203/rs.3.rs-21206/v1

64. Marak BN, Dowarah J, Khiangte L, Singh VP (2021) Step toward repurposing drug discovery for COVID-19 therapeutics through in silico approach. Drug Dev Res 82(3):374-392

65. Sotoudeh E, Sotoudeh H (2020) A hypothesis about the role of fetal hemoglobin in COVID-19. Med Hypotheses 144(1):109994

66. Esposito G, Pesce M, Seguella L, Sanseverino W, Lu J, Corpetti C, Sarnelli $\mathrm{G}$ (2020) The potential of cannabidiol in the COVID-19 pandemic. Br J Pharmacol 177(21):4967-4970

67. Hayakawa J (2021) Favipiravir/loxoprofen/paracetamol: symmetrical drug-related intertriginous and flexural exanthema: case report. React Wkly 1841(1):113

68. Siminea N, Popescu V, Martin JAS, Florea D, Gavril G, Gheorghe A-M, Itcus C, Kanhaiya K, Pacioglu O, Popa LI, et al. (2021) Network analytics for drug repurposing in COVID-19. arXiv preprint arXiv:2106.12297

69. Li F, Michelson AP, Foraker R, Zhan M, Payne PR (2020) Repurposing drugs for COVID-1 9 based on transcriptional response of host cells to SARSCOV-2. arXiv e-prints, 2006

70. Hashimoto K (2021) Repurposing of CNS drugs to treat COVID-19 infection: targeting the sigma-1 receptor. Eur Arch Psychiatry Clin Neurosci $1(1): 1-10$

71. Nobile B, Durand M, Olié E, Guillaume S, Molès J, Haffen E, Courtet $P$ (2020) Clomipramine could be useful in preventing neurological complications of SARS-COV-2 infection. J Neuroimmune Pharmacol 15(3):347-348

72. Marinella MA (2020) Indomethacin and resveratrol as potential treatment adjuncts for SARS-COV-2/COVID-19. Int J Clin Pract 74(9):13535

73. Miryan M, Bagherniya M, Sahebkar A, Soleimani D, Rouhani MH, Iraj B, Askari G (2020) Effects of curcumin-piperine co-supplementation on clinical signs, duration, severity, and inflammatory factors in patients with COVID-19: a structured summary of a study protocol for a randomised controlled trial. Trials 21(1):1-2

74. Soni VK, Mehta A, Ratre YK, Tiwari AK, Amit A, Singh RP, Sonkar SC, Chaturvedi N, Shukla D, Vishvakarma NK (2020) Curcumin, a traditional spice component, can hold the promise against COVID-19? Eur J Pharmacol 886(1):173551

75. Sestili P, Fimognari C (2020) Paracetamol-induced glutathione consumption: is there a link with severe COVID-19 illness? Front Pharmacol 11(1):1597 